Received: 2020-11-21

Accepted: 2020-12-06

Published: 2020-12-08

\section{Online exercise rehabilitation to stable COPD patients during the second COVID wave: Are physiotherapists able to help?}

\author{
Ali Mohamed Ali Ismail \\ Department of Physical Therapy for Cardiovascular/Respiratory Disorder \\ and Geriatrics, Faculty of Physical Therapy, Cairo University, Giza, Egypt
}

Correspondence: Ali Mohamed Ali Ismail; Department of Physical Therapy for Cardiovascular/Respiratory Disorder and Geriatrics, Faculty of Physical Therapy, Cairo University, Giza, Egypt; email: ali.mohamed@pt.cu.edu.eg

\section{Dear Editor,}

A significant cause of morbidity and mortality worldwide is a chronic obstructive pulmonary disease (COPD). It is a chronic lung condition characterized by gradual restriction of airflow and symptomatic respiratory complaints, such as worsening dyspnea, chronic cough, and sputum. Besides the frequent symptomatic exacerbations (that need hospitalization), COPD may contribute to the gradual intolerance of exercise that eventually reduces the quality of life (QoL) of patients [1]. Reduced QoL in COPD patients may be related to the gradual dysfunction of peripheral limb and respiratory muscles that restrict the daily activities [2].

With a relatively-mild first wave from March to July 2020, the outbreak of the corona virus disease 2019 (COVID-19) spread to all countries around the world. The strong specter of the second wave began to appear from August 2020 till now [3] with the return of precautionary measures such as social distancing enforced by some governments, especially in Europe, reaching to some Arab countries including Egypt, which is on the threshold of the second wave of COVID-19, but more fiercely than the first wave, especially with the arrival of winter which represents a great opportunity for COVID-19 transmission.

During the endless incurable COVID pandemic, continuous stable-COPD assessment, treatment, and follow-up from the whole medical team (including chest physicians, psychologists, nutritionists, and physiotherapists) are recommended utilizing the telemedicine technologies away from the traditional face-to-face meetings to lower the high risk of COVID-19 contamination [4]. Maintaining a moderate reasonable level of physical activity is a very important factor to decline the infection risk to the respiratory tract in addition to overcoming the expected physical and psychological adverse effects [5] of intentional/forced social distance even in patients with stable chronic long-standing chest diseases who are more vulnerable to high rate of deaths due to the cross-COVID risk infection.

With the repeated calls to physiotherapists - during the first wave of COVID-19 crisis - to participate in the online home-centered rehabilitation sessions to avoid the expected high rate of patients -especially the elder ones - who will (un)intentionally cancel the continuity of their rehabilitation sessions in the private and/or governmental physiotherapy units that may be locked down due to the formal recommendations to cancel the not urgent long-timed treatment services during the pandemic COVID-19 [6].

During the second-COVID wave, remotely-supervised online exercise rehabilitation by physiotherapists is one of the available and recommended home options to 
continue the demanded exercise rehabilitation to avoid the high COVID-cross risk infection that may be transmitted to low-immunity stable COPD patients attended to physiotherapy centers. Physiotherapists - under the supervision of physiotherapy syndicates in Egypt must write a simple foldout - in native language - that contains the different components of exercise rehabilitation that can be simply carried out by the stable COPD patients at home, starting from different breathing exercises aiming to improve relaxation, strengthen of respiratory muscles (as a diaphragmatic, pursed-lip, postureconnected breathing, and belt breathing exercises), and prevent the accumulation of airway secretions (forced expiration techniques, active cycle of breathing, and autogenic drainage). Simple active free and resisted strengthening exercises for the muscles of upper and lower extremities must be incorporated in the foldout. Electronic copies of the foldout may be emailed or sent to the different social media accounts of every patient.

It is very important to supply the patients and/or family caregivers with the available websites for selecting, booking, online-ordering, and home-delivery of different respiratory training devices (flow-ball lungentrainer, breath builder classic, and AirPhysio Sports) that add a fun-encouraging element to some geriatric COPD patients who may be bored during the routine application of the above-mentioned breathing and limb exercises. Also, it is crucial to guide the patients and/or family members to other respiratory retraining devices as the Airofit Breathing Trainer device that enables the patient to monitor his/her lung capacities via the associated-mobile application with the device.

It is very urgent to construct videos - by physiotherapists - containing how to apply the self-rehabilitation sessions via the above-mentioned respiratory retraining devices, breathing exercises, and limb stretching and strengthening exercises. The foldout and videos may be uploaded - with the help of the main national physiotherapy syndicate - on the official national website and Facebook page of the ministry of health. Regular 3-timed scheduled meetings per week on the free videoconferencing applications as zoom may be adopted by physiotherapists as a way to maintain the supervision of exercise rehabilitation in stable COPD patients.

Finally, some stable COPD patients - who prefer the assistance of mobile health applications that introduce monitoring of vital signs during home exercise rehabilitation via application-associated wearable monitoring accessories - may be guided from physiotherapists to different websites of paid rehabilitation applications as mHealth mobile application that may be downloaded on their cell phones to maintain their physical activity at home during the second COVID wave.

\section{Conflicts of interest}

The authors declare no conflict of interest.

\section{References}

1. Wu ZY, Han YX, Niu ME, et al. Handgrip strength is associated with dyspnoea and functional exercise capacity in male patients with stable COPD. Int J Tuberc Lung Dis. 2019; 23(4): 428-32.)

2. Tymruk-Skoropad K, Tsizh L, Vynogradskyi B, Pavlova I. Physical therapy in chronic obstructive pulmonary disease (analysis of the evidence-based medicine). Physiother Q. 2018; 26(2): 1-8.

3. Horton R. Offline: The second wave. Lancet (London, England) 2020; 395(10242); 1960 .

4. Deslée G, Zysman M, Burgel PR, et al. Chronic obstructive pulmonary disease and the COVID-19 pandemic: reciprocal challenges. Respir Med Res. 2020; 78: 100764.

5. Hull JH, Loosemore M, Schwellnus M. Respiratory health in athletes: facing the COVID-19 challenge. Lancet Resp Med. 2020; 8(6): 557-8.

6. Ismail AMA. Cancelled elderly exercise sessions during the COVID-19 crisis: can physical therapists help from their homes? Eur J Physiother. 2020; 22(4): 235. 